Research Paper

\title{
Superior prognosis stratification for stage III colon cancer using log odds of positive lymph nodes (LODDS) compared to TNM stage classification: the Japanese study group for postoperative follow-up of colorectal cancer
}

\author{
Shimpei Ogawa ${ }^{1}$, Michio Itabashi ${ }^{1}$, Yoshiko Bamba ${ }^{1}$, Masakazu Yamamoto ${ }^{1}$ and \\ Kenichi Sugihara ${ }^{2}$ \\ ${ }^{1}$ Department of Surgery, Institute of Gastroenterology, Tokyo Women's Medical University School of Medicine, Tokyo, Japan \\ ${ }^{2}$ Department of Surgery, Tokyo Medical and Dental University, Tokyo, Japan \\ Correspondence to: Shimpei Ogawa, email: ogawa.shimpei@twmu.ac.jp \\ Keywords: colon cancer; LODDS; TNM; staging; prognosis \\ Received: April 25, $2020 \quad$ Accepted: July 14, $2020 \quad$ Published: August 18, 2020
}

Copyright: Ogawa et al. This is an open-access article distributed under the terms of the Creative Commons Attribution License 3.0 (CC BY 3.0), which permits unrestricted use, distribution, and reproduction in any medium, provided the original author and source are credited.

\section{ABSTRACT}

Objectives: The aim of this study is to examine whether prognosis stratification in staging of Stage III colon cancer using T factor and log odds of positive lymph nodes (LODDS) categories is superior to that of the TNM staging system.

Materials and Methods: The subjects were 5,919 patients with Stage III colon cancer who underwent curative resection at 24 Japanese institutions. Univariate analysis of LODDS categories and clinicopathologic factors was conducted using a Cox proportional hazards regression model for cancer-specific survival (CSS). Independent prognostic factors for CSS were extracted in multivariate analysis using factors with significance in univariate analysis. Effect sizes of risk factors for CSS were compared using the LogWorth statistic. Combinations of $T$ factor and LODDS categories were used to create L-stage subgroups A, B and C. Stratification of prognosis with L-stage and TNM was compared using the Akaike information criterion (AIC).

Results: In multivariate analysis, LODDS was identified as an independent prognostic factor, together with age, maximum tumor diameter, histopathological grade, L, V, PT, and pN. The LogWorth of LODDS was 17.149, which was the second highest after pT (31.562), and that of pN was 7.434. The 5-year CSS was $96.5 \%$, $88.5 \%$, and $66.6 \%$ in TNM stages IIIA, IIIB, and IIIC, respectively, and $96.0 \%$, $87.6 \%$, and $59.3 \%$ in L-stage A, B, and C, respectively $(p<0.0001)$. AICs for TNM and L-stage were $14,795.5$ and $14,707.8$, respectively.

Conclusions: Prognosis stratification of the stage classification for Stage III colon cancer was superior with L-stage compared to TNM stage classification.

\section{INTRODUCTION}

Staging of colon cancer is widely used as an indicator for selection of therapeutic strategy and prognostic prediction. The TNM staging system of the American Joint Committee on Cancer (AJCC) and the Union International Cancer Control (UICC) is the most widely used worldwide [1]. Lymph node metastasis (LNM) is an important prognostic factor in this system $[2,3]$. The $\mathrm{N}$ grade in TNM staging is determined based on the number of LNMs, but does not reflect the number of lymph node dissections (LNDs). However, this number may also be a prognostic factor in colon cancer, with more LNDs suggesting a more favorable prognosis [4-6]. More LNDs also reduces stage migration and enables correct grading $[7,8]$. Therefore, the number of LNDs may have a large impact on grading of the $\mathrm{N}$ category in TMN staging.

The lymph node ratio (LNR) and the log odds of positive lymph nodes (LODDS) are used to reflect the numbers of LNDs and LNMs in staging [9, 10]. LODDS is calculated based on the numbers of lymph nodes that are positive and negative for metastasis, and has been reported 
to be useful for selection of high-risk patients, prognosis prediction, and stratification for patients with breast, gastric, and colorectal cancers [2, 11-15]. In TNM staging, Stage III colon cancer is stratified into subgroups: Stage IIIA, Stage IIIB, and Stage IIIC in T and N categories. A more precise staging system may be possible using LODDS, in which the number of LNDs is added instead of the $\mathrm{N}$ category, but this has not been examined to date. Therefore, the aim of this study is to examine whether a staging system for Stage III colon cancer based on the $\mathrm{T}$ category and LODDS enables stratification of patients to give a homogeneous and more accurate prognosis, compared to TNM staging.

\section{RESULTS}

\section{LODDS category}

The mean numbers of LNDs and LNMs in the subjects were $24.5 \pm 14.8$ and $2.89 \pm 3.13$, respectively. Three cut-off points were extracted based on classification and regression trees (CART) analysis (-1.711, -0.717, $0.077)$. LODDS was classified into four categories: $\mathrm{A} \leq$ $-1.711 ; \mathrm{B}>-1.711$ to $\leq-0.717 ; \mathrm{C}>-0.717$ to $\leq 0.077$; and $\mathrm{D}>0.077$, which included 3648, 1604, 459 and 208 patients, respectively (Table 1).

\section{Risk factors}

Among the 5,919 subjects, 908 (15.3\%) had cancerspecific deaths. Cox proportional hazards regression analysis was used to identify factors associated with cancer-specific survival (CSS). In univariate analysis, gender, maximum tumor diameter, histopathological grade, lymphatic invasion (L), venous invasion (V), pathologic $\mathrm{T}$ stage $(\mathrm{pT})$, pathologic $\mathrm{N}$ stage $(\mathrm{pN})$ and LODDS category were significantly related to CSS. In multivariate analysis, male gender, maximum tumor diameter $(\geq 41.0 \mathrm{~mm})$, histopathological grade (G3 + G4), L1, V1, pT (T3, T4a, T4b), pN (N1b, N2a, N2b) and LODDS (B, C, D) were independent factors associated with CSS (Table 2). The LogWorth for pT of 31.562 was highest, followed by 17.149 for LODDS category and 7.434 of $\mathrm{pN}$, suggesting that the LODDS category was more important than $\mathrm{pN}$ (Figure 1).

\section{L-staging}

Twenty combinations of pT and LODDS category were used to establish three subgroups (L-stage A, B and C) based on CSS. Combinations of T1 and LODDS A, B, and $\mathrm{C}$; T2 and LODDS A, B, and C; and T3 and LODDS A, which had CSS $\geq 90 \%$, were included in L-stage A. Combinations of T1 and LODDS D; T2 and LODDS D; T3 and LODDS B and C; T4a and LODDS A and B; and T4b and LODDS A, which had CSS $\geq 70 \%$ to $<90 \%$, were included in L-stage B. Other combinations were included in L-stage C (Figure 2A and 2B).

The 5-year CSS was $96.5 \%, 88.5 \%$, and $66.6 \%$ in TNM stages IIIA, IIIB, and IIIC, respectively $(p<$ 0.0001 ), and $96.0 \%, 87.6 \%$, and $59.3 \%$ in L-stages A, B, and $\mathrm{C}$, respectively $(p<0.0001)$. The Akaike information criterion (AIC) values for TNM stage and L-stage were $14,795.5$ and 14,707.8, respectively, with a lower value for L-stage (Figure 3A and 3B).

\section{DISCUSSION}

The lower AIC value for L-staging for Stage III colon cancer, based on $\mathrm{pT}$ and LODDS categories, compared to that for TNM staging, suggests that stratification using L-staging is superior to that with TNM staging. This may be due to the impact of the LODDS category. In multivariate analysis, $\mathrm{pT}, \mathrm{pN}$, and LODDS category were independently associated with CSS, in addition to gender, maximum tumor diameter, histopathological grade, L, and V. The highest LogWorth of 31.562 was found for $\mathrm{pT}$, followed by 17.149 for LODDS category and 7.434 for $\mathrm{pN}$. Logworth is calculated as $-\log _{10}$ ( $p$ value), and higher values are considered to be more significant [16]. In this study, $\mathrm{pT}$, LODDS, and $\mathrm{pN}$ were extracted as independent risk factors for CSS in multivariate analysis, with $P<0.0001$ for each factor. Thus, the individual impact of each risk factor for CSS could not be compared based on the $P$ value, but LogWorth values could be used for this comparison. Thus, the higher LogWorth for the LODDS category compared to the $\mathrm{N}$ category suggests the importance of the LODDS category in stratification of CSS. Thus, cases with a poor prognosis can be selected using the LODDS category. The hazard ratio (HR) of N2b vs. N1a in $\mathrm{pN}$ was 2.29, whereas HR for LODDS D vs. A was 3.57, which also indicates that cases with a poor prognosis are better identified using the LODDS category.

The number of LNDs in patients with colon cancer depends on surgical technique, quality of histopathological examination, tumor biology, and patient-tumor immunologic response [17-20]. More LNDs has been associated with a more favorable prognosis in LNMpositive and LNM-negative cases [4-6]. This may be because high-quality surgery results in appropriate and probably increased LNDs. In addition, micrometastases and isolated tumor cells, which are difficult to identify in routine pathological examinations, may be removed by LND [7, 21]. A high-quality histopathological examinations allows accurate evaluation of the $\mathrm{N}$ category, and this reduces the number of underestimated and missed cases for which adjuvant therapy is required, which is also likely to improve the therapeutic outcome. These findings show that the number of LNDs is an important factor that should be taken into consideration in stratification and prediction of outcome in N staging. Since LODDS is calculated based on the numbers of negative and positive LNMs and LNDs, staging is reflected more accurately. 
Table 1: Clinicopathologic characteristics of patients

\begin{tabular}{|c|c|}
\hline Characteristic & Value \\
\hline Age (median; range) & $67(19-99)$ \\
\hline \multicolumn{2}{|l|}{ Gender } \\
\hline Male & $3163(53.4)$ \\
\hline Female & $2756(46.6)$ \\
\hline Maximum tumor diameter (median; range) & $41.0(11-135)$ \\
\hline \multicolumn{2}{|l|}{ Histopathological grade } \\
\hline $\mathrm{G} 1, \mathrm{G} 2$ & $5385(91.0)$ \\
\hline G3, G4 & $534(9.0)$ \\
\hline \multicolumn{2}{|l|}{ Lymphatic invasion } \\
\hline L0 & $1268(21.4)$ \\
\hline L1 & $4651(78.6)$ \\
\hline \multicolumn{2}{|l|}{ Venous invasion } \\
\hline V0 & $1526(25.8)$ \\
\hline $\mathrm{V} 1$ & $4393(74.2)$ \\
\hline \multicolumn{2}{|l|}{ Postoperative adjuvant therapy } \\
\hline No & $2246(37.9)$ \\
\hline Yes & $3673(62.1)$ \\
\hline \multicolumn{2}{|l|}{ Number of retrieved lymph nodes } \\
\hline$<12$ & $966(16.3)$ \\
\hline$\geq 12$ & $4953(83.7)$ \\
\hline \multicolumn{2}{|l|}{ Pathologic $T$ stage } \\
\hline $\mathrm{T} 1$ & $285(4.8)$ \\
\hline $\mathrm{T} 2$ & $510(8.6)$ \\
\hline $\mathrm{T} 3$ & $3456(58.4)$ \\
\hline $\mathrm{T} 4 \mathrm{a}$ & $1307(22.1)$ \\
\hline $\mathrm{T} 4 \mathrm{~b}$ & $361(6.1)$ \\
\hline \multicolumn{2}{|l|}{ Pathologic $N$ stage } \\
\hline N1a & $2437(41.2)$ \\
\hline N1b & $2002(33.8)$ \\
\hline $\mathrm{N} 2 \mathrm{a}$ & $1005(17.0)$ \\
\hline $\mathrm{N} 2 \mathrm{~b}$ & $475(8.0)$ \\
\hline \multicolumn{2}{|l|}{ LODDS category } \\
\hline LODDS A & 3648 (61.6) \\
\hline LODDS B & $1604(27.1)$ \\
\hline LODDS C & $459(7.8)$ \\
\hline LODDS D & $208(3.5)$ \\
\hline
\end{tabular}

LNR is also used as an indicator that includes the number of LNDs in N staging $[9,10]$. LNR is calculated by dividing LNMs by LNDs, and is a useful prognostic factor in colon cancer. Ceelen et al. [10] found that LNR was an independent prognostic factor for Stage III colon cancer in a meta-analyses of 16 studies. However, there are problems with use of LNR. For example, if all LNDs are positive for metastasis, LNR is equal to 1, regardless of the total number of LNDs, and all cases with LNR of 1 are classified in the same category. However, prognoses can differ based on the total numbers of LNMs and LNDs.

In contrast, in LODDS, 0.5 is added to the number of LNMs and to the number of lymph nodes negative for metastasis, and thus the value differs depending on the number of LNMs, even when the numbers of LNMs and LNDs are the same. For example, LODDS is 
Table 2: Univariate and multivariate analysis of risk factors for cancer-specific survival

\begin{tabular}{|c|c|c|c|c|c|c|c|}
\hline \multirow[t]{2}{*}{ Factor } & \multirow{2}{*}{$\begin{array}{c}\text { Number of } \\
\text { patients }(\%)\end{array}$} & \multicolumn{3}{|c|}{ Univariate analysis } & \multicolumn{3}{|c|}{ Multivariate analysis } \\
\hline & & Hazard ratio & $95 \% \mathrm{CI}$ & $P$ & Hazard ratio & $95 \% \mathrm{CI}$ & $P$ \\
\hline \multicolumn{8}{|l|}{ Age } \\
\hline$<68$ & $3012(50.9)$ & 1 & & & & & \\
\hline$\geq 68$ & $2907(49.1)$ & 1.13 & $0.99-1.29$ & 0.0682 & & & \\
\hline \multicolumn{8}{|l|}{ Gender } \\
\hline Male & $3163(53.4)$ & 1.14 & $1.00-1.30$ & 0.0499 & 1.15 & $1.00-1.31$ & 0.0423 \\
\hline Female & $2756(46.6)$ & 1 & & & 1 & & \\
\hline \multicolumn{8}{|c|}{ Maximum tumor diameter } \\
\hline$<41.0$ & $2947(49.8)$ & 1 & & & 1 & & \\
\hline$\geq 41.0$ & $2972(50.2)$ & 1.49 & $1.31-1.70$ & $<0.0001$ & 1.16 & $1.01-1.33$ & 0.0347 \\
\hline \multicolumn{8}{|c|}{ Histopathological grade } \\
\hline $\mathrm{G} 1, \mathrm{G} 2$ & $5385(91.0)$ & 1 & & & 1 & & \\
\hline G3, G4 & $534(9.0)$ & 1.93 & $1.59-2.31$ & $<0.0001$ & 1.41 & $1.16-1.70$ & 0.0007 \\
\hline \multicolumn{8}{|c|}{ Lymphatic invasion } \\
\hline L0 & $1268(21.4)$ & 1 & & & 1 & & \\
\hline L1 & $4651(78.6)$ & 1.90 & $1.58-2.31$ & $<0.0001$ & 1.33 & $1.10-1.62$ & 0.0034 \\
\hline \multicolumn{8}{|c|}{ Venous invasion } \\
\hline V0 & $1526(25.8)$ & 1 & & & 1 & & \\
\hline V1 & $4393(74.2)$ & 1.68 & $1.42-2.00$ & $<0.0001$ & 1.22 & $1.03-1.46$ & 0.0200 \\
\hline \multicolumn{8}{|c|}{ Postoperative adjuvant therapy } \\
\hline No & $2246(37.9)$ & 1.07 & $0.93-1.22$ & 0.3296 & & & \\
\hline Yes & $3673(62.1)$ & 1 & & & & & \\
\hline \multicolumn{8}{|c|}{ Number of retrieved lymph nodes } \\
\hline$<12$ & $966(16.3)$ & 1 & & & & & \\
\hline$\geq 12$ & $4953(83.7)$ & 1.04 & $0.87-1.26$ & 0.6485 & & & \\
\hline \multicolumn{8}{|c|}{ Pathologic T stage } \\
\hline $\mathrm{T} 1$ & $285(4.8)$ & 1 & & & 1 & & \\
\hline $\mathrm{T} 2$ & $510(8.6)$ & 2.06 & $0.98-4.32$ & 0.0418 & 1.79 & $0.85-3.75$ & 0.1066 \\
\hline $\mathrm{T} 3$ & $3456(58.4)$ & 4.52 & $2.34-8.75$ & $<0.0001$ & 3.32 & $1.70-6.46$ & $<0.0001$ \\
\hline $\mathrm{T} 4 \mathrm{a}$ & $1307(22.1)$ & 9.57 & $4.93-18.57$ & $<0.0001$ & 5.94 & $3.03-11.63$ & $<0.0001$ \\
\hline $\mathrm{T} 4 \mathrm{~b}$ & $361(6.1)$ & 14.54 & $7.38-28.67$ & $<0.0001$ & 9.09 & $4.55-18.16$ & $<0.0001$ \\
\hline \multicolumn{8}{|c|}{ Pathologic N stage } \\
\hline N1a & $2437(41.2)$ & 1 & & & 1 & & \\
\hline $\mathrm{N} 1 \mathrm{~b}$ & $2002(33.8)$ & 1.65 & $1.39-1.96$ & $<0.0001$ & 1.37 & $1.14-1.64$ & 0.0008 \\
\hline $\mathrm{N} 2 \mathrm{a}$ & $1005(17.0)$ & 2.44 & $2.02-2.94$ & $<0.0001$ & 1.56 & $1.25-1.95$ & $<0.0001$ \\
\hline $\mathrm{N} 2 \mathrm{~b}$ & $475(8.0)$ & 5.57 & $4.57-6.77$ & $<0.0001$ & 2.29 & $1.76-2.99$ & $<0.0001$ \\
\hline \multicolumn{8}{|l|}{ LODDS } \\
\hline LODDS A & 3648 (61.6) & 1 & & & 1 & & \\
\hline LODDS B & $1604(27.1)$ & 1.70 & $1,46-1.97$ & $<0.0001$ & 1.29 & $1.08-1.54$ & 0.0054 \\
\hline LODDS C & $459(7.8)$ & 2.69 & $2.18-3.30$ & $<0.0001$ & 1.69 & $1.31-2.17$ & $<0.0001$ \\
\hline LODDS D & $208(3.5)$ & 6.99 & $5.59-8.67$ & $<0.0001$ & 3.57 & $2.70-4.71$ & $<0.0001$ \\
\hline
\end{tabular}

Abbreviations, CI: confidence interval.

1.099 for a case with 1 LND and 1 LNM, but 2.708 for a case with 7 LNDs and 7 LNMs. In addition, since the difference becomes larger in cases with a smaller number of LNMs (that is, cases with fewer LNDs), stratification can be performed for these cases. In fact, cases with $<12$ LNDs may not be appropriately stratified by LNR, but with LODDS such cases can be stratified [9, 22-24]. In addition, in LNM-negative Stage I or II cases, LNR is 0 regardless of the number of LNDs, but with LODDS, the value differs depending on the number of LNDs [2, 25]. These properties show the utility of LODDS as a prognostic factor and in $\mathrm{N}$ staging. 
The current study suggests that L-staging can be performed using LODDS. The AIC value was lower than that for TNM staging, and thus L-staging may better stratify the prognosis of patients with Stage III colon cancer. The 5-year CSS was almost the same in TNM stage IIIA and L-stage A, and in TNM stage IIIB and L-stage B, but differed between TNM stage IIIC $(66.6 \%)$ and L-stage $C(59.3 \%)$. This suggests that cases with a poor prognosis may be better identified using the L-staging system. Postoperative adjuvant therapy for Stage III colon cancer is not uniform, and various drugs and administration periods are used. To select this therapy, accurate stratification of stage is required, and the L-staging system enables more accurate stratification, compared to TNM staging. This suggests that L-staging could contribute to planning of optimum regimens of drugs and administration periods for individual patients.

This study has some limitations. It was a retrospective study of cases in high-volume centers in Japan, and further cases are needed for prospective analysis. The chosen cutoff value in the LODDS category classification has a large impact on stratification by L-staging. Cutoffs were calculated using CART, but more appropriate cutoffs may further improve the L-staging system. It is also unclear if this system can be used for Stage I or II cases, including those with preoperative treatment, rectal cancer, many dissected lymph nodes, and a LNM-negative status, which were excluded from this study.

\section{MATERIALS AND METHODS}

\section{Patients}

The subjects were 5,919 patients with Stage III colon cancer (excluding appendiceal cancer) who underwent curative resection between January 1997 and December 2012 at 24 Japanese institutions, all of which were in the Japanese Study Group for Postoperative Follow-up of Colorectal Cancer (JFUP-CRC). No patients received neoadjuvant therapy. The inclusion criteria were a pathological diagnosis of colon cancer, complete clinicopathological factors (age, gender, histopathological grade, maximum tumor diameter, $\mathrm{L}, \mathrm{V}, \mathrm{pT}, \mathrm{pN}$, number of lymph nodes analyzed, number of positive lymph nodes, postoperative adjuvant treatment), and time and vital status at the last follow-up clearly noted. Stages are reported using the TNM classification in the UICC Staging Manual. This study was approved by the Central Institutional Review board (Tokyo Medical and Dental University) and local ethical committee.

\section{LODDS}

LODDS values are defined as $\log ([\mathrm{pLN}+0.5] /$ $[\mathrm{nLN}+0.5])$, where $\mathrm{pLN}$ and $\mathrm{nLN}$ are the numbers of positive and negative lymph nodes, respectively. A value of 0.5 is added to the numerator and denominator to avoid a singularity error. To investigate optimal categorization of LODDS, CART was used to determine highly discriminating cutoffs for CSS, using the R software package ver. 3.5.3 (The R Foundation for Statistical Computing Platform: x86_64-w64-mingw32/x64). CSS was defined as time from surgery to death due to cancer recurrence. Based on three obtained cutoffs, LODDS was divided into four categories (A, B, C, D).

\section{Risk factors}

Univariate analysis of LODDS category and other clinicopathologic factors was conducted using a Cox proportional hazards regression model for CSS. Independent prognostic factors for CSS were extracted based in multivariate analysis using factors with significance in univariate analysis. Effect sizes of independent factors on

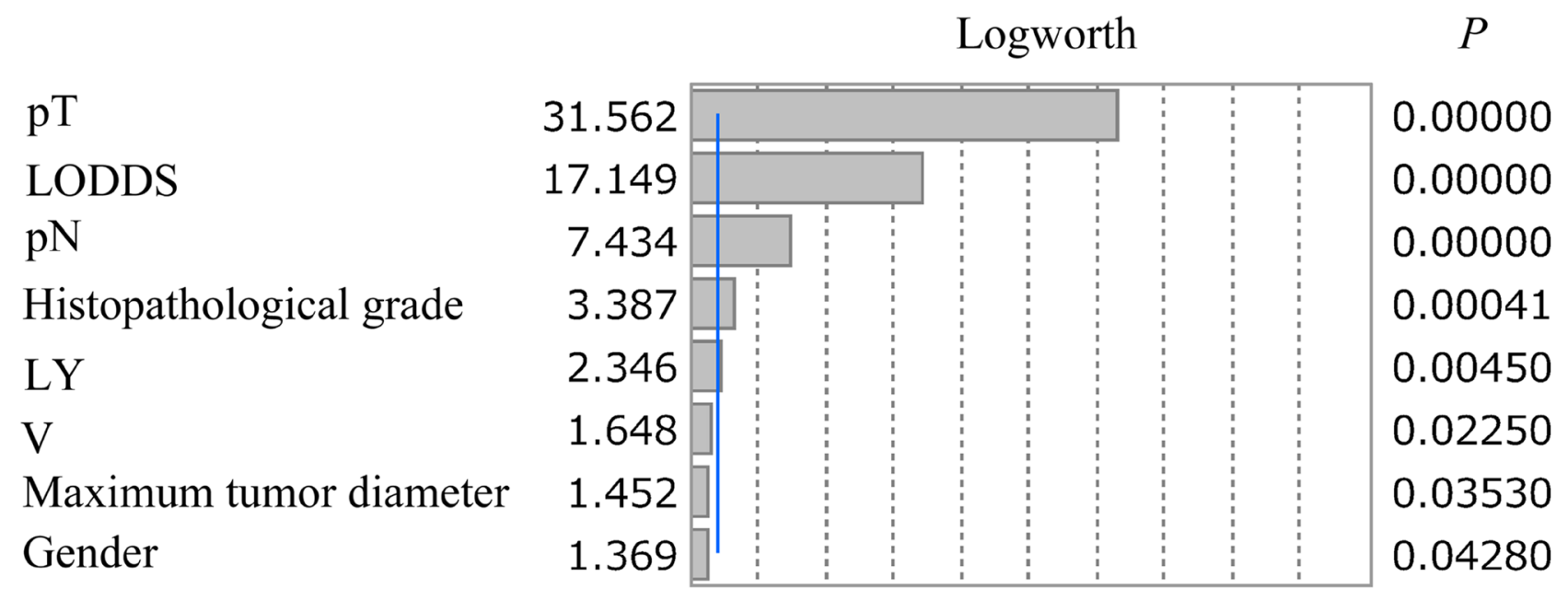

Figure 1: LogWorth of independent risk factors identified in multivariate analysis. The LogWorth of pT was 31.562 , followed by 17.149 for LODDS category and 7.434 for $\mathrm{pN}$. The blue line indicates a LogWorth of 2 . 


\section{A}

\begin{tabular}{|c|c|c|c|c|}
\hline & LODDS A & LODDS B & LODDS C & LODDS D \\
\hline $\mathrm{T} 1$ & $97.7 \%$ & $95.7 \%$ & $100 \%$ & $83.3 \%$ \\
\hline $\mathrm{T} 2$ & $95.8 \%$ & $93.6 \%$ & $92.0 \%$ & $87.5 \%$ \\
\hline $\mathrm{T} 3$ & $91.7 \%$ & $87.6 \%$ & $79.2 \%$ & $45.6 \%$ \\
\hline $\mathrm{T} 4 \mathrm{a}$ & $83.2 \%$ & $75.9 \%$ & $56.1 \%$ & $43.2 \%$ \\
\hline $\mathrm{T} 4 \mathrm{~b}$ & $75.4 \%$ & $56.7 \%$ & $58.6 \%$ & $30.3 \%$ \\
\hline
\end{tabular}

B

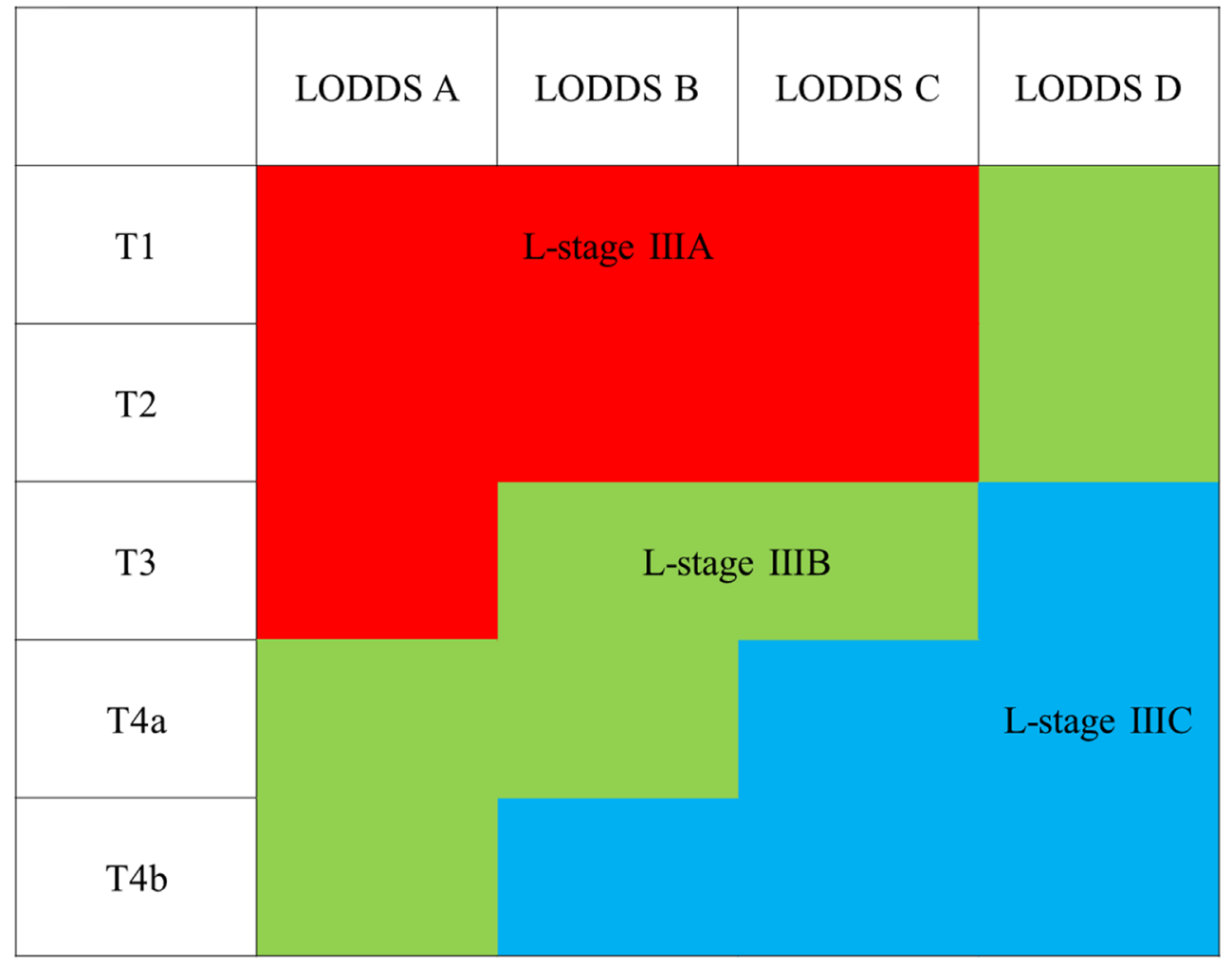

Figure 2: Classification of L-stage subgroups based on 5-year cancer specific survival (CSS). (A) 5-year CSS in pathologic $\mathrm{T}$ and LODDS category. (B) L-stage IIIA: 5-year CSS $\geq 90 \%$ (red), L-stage IIIB: 5-year CSS $\geq 70 \%$ to $<90 \%$ (green), and L-stage IIIC: 5 -year CSS $<70 \%$ (blue). 
CSS were compared using LogWorth (where LogWorth is $-\log 10$ [ $p$-value], such that $p=0.01$ is equivalent to a $\log$ Worth of 2.0, $p=0.001$ is denoted by a LogWorth of 3.0 , etc.) for further interpretative clarity [16].

\section{Construction of the L-staging system}

Twenty combinations of T category (T1, T2, T3, T4a, T4b) and LODDS category (A, B, C, D) were divided into 3 subgroups based on CSS to construct a L-staging system.

\section{Statistical analysis}

Survival curves were generated using the KaplanMeier method and compared by log-rank test. Comparison of stratification of survival outcomes using TNM stage and L-stage was performed using the AIC calculated in a Cox proportional hazards regression model to identify the better system for predicting outcomes. A lower AIC value was considered to be optimal. In each analysis, $P<0.05$ was taken to be significant. All analyses were performed

A

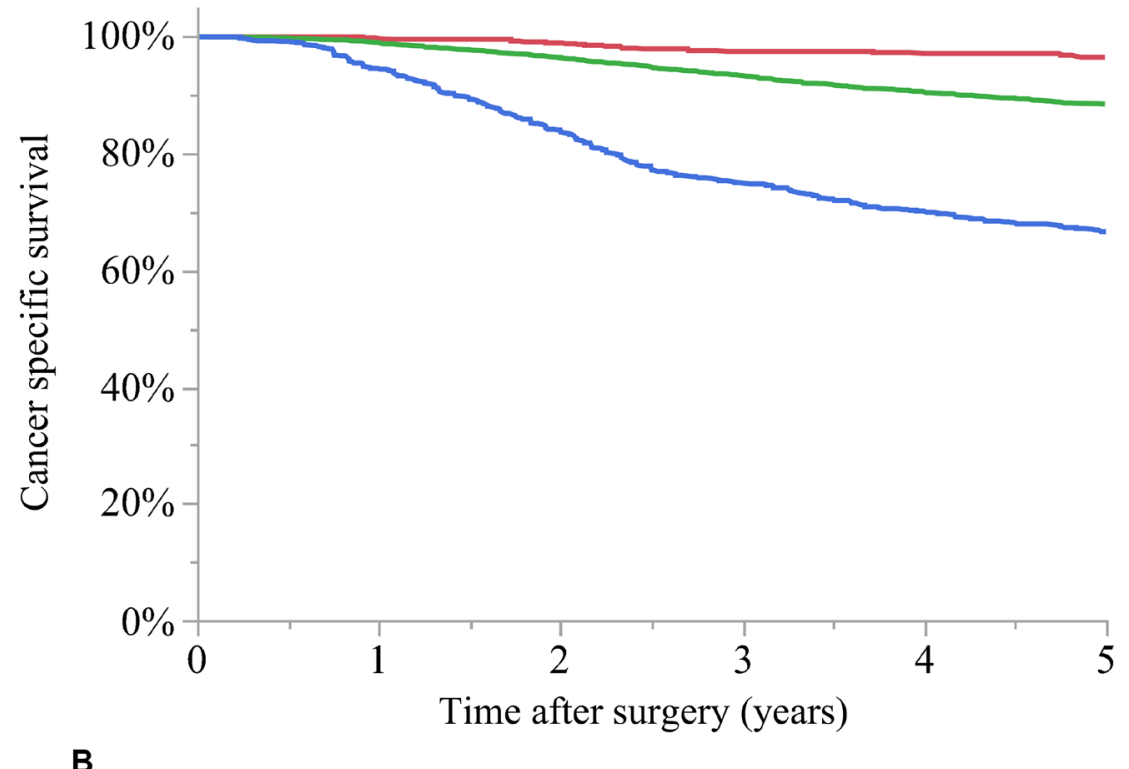

B

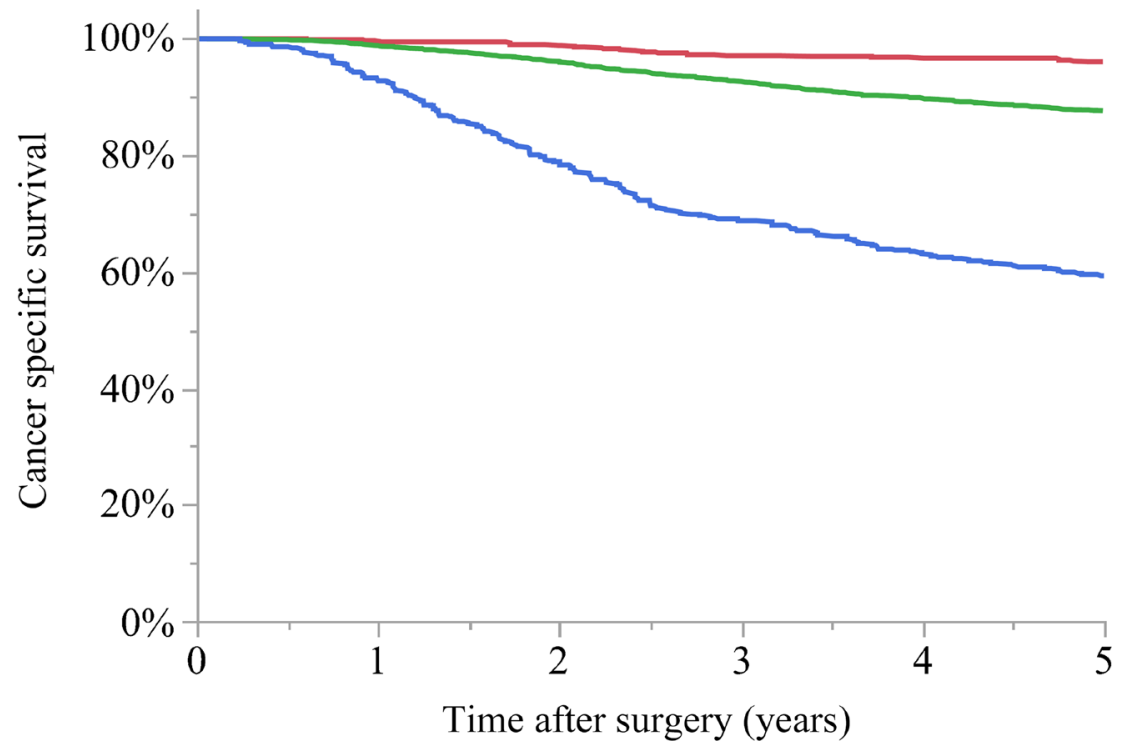

Figure 3: Cancer-specific survival (CSS) curves for stage III colon cancer. (A) TNM stage: 5-year CSS for stage IIIA (red): 96.5\%, stage IIIB (green): $88.5 \%$, and stage IIIC (blue): 66.6\% ( $p<0.0001)$. (B) L-stage: 5-year CSS for A (red): $96.0 \%$, B (green): $87.6 \%$, and C (blue): $59.3 \%(p<0.0001)$. The Akaike information criterion value was 14,795.5 for TNM stage and 14,707.8 for L-stage. 
using JMP Pro ver.14 for Windows ${ }^{\circledR}$ (SAS Institute Inc., Cary, NC, USA).

\section{CONCLUSIONS}

Our results suggest that a staging system using LODDS for Stage III colon cancer may stratify prognosis more accurately than the TNM staging system. This is important because accurate stratification of prognosis will enable individual adjustment of treatments appropriate for the estimated prognosis, which should lead to a better therapeutic outcome.

\section{Abbreviations}

AJCC: American Joint Committee on Cancer; UICC: Union for International Cancer Control; LNM: lymph node metastasis; LND: lymph node dissection; LNR: lymph node ratio; LODDS: log odds of positive lymph nodes; CART: classification and regression trees; CSS: cancer-specific survival; L: lymphatic invasion; V: venous invasion; $\mathrm{pT}$ : pathologic T stage; $\mathrm{pN}$ : pathologic N stage; AIC: Akaike information criterion; HR: hazard ratio; JFUP-CRC: Japanese Study Group for Postoperative Follow-up of Colorectal Cancer.

\section{Author contributions}

Study concepts: S.O, M.I, Y.B, M.Y and K.S. Study design: S.O and M.I. Data acquisition: S.O and Y.B. Data analysis and interpretation: S.O, M.I and Y.B. Revising of the manuscript: S.O, M.I, Y.B and M.Y. Final manuscript approval: S.O, M.I, Y.B, M.Y and K.S.

\section{ACKNOWLEDGMENTS}

This study was based on data from the following 24 referral institutions in the Japanese Study Group for Postoperative Follow-up of Colorectal Cancer: I. Takemasa (Sapporo Medical University), K. Hakamada (Hirosaki University), H. Kameyama (Niigata University), Y. Takii (Niigata Cancer Center Hospital), H. Ueno (National Defense Medical College), H. Ozawa (Tochigi Cancer Center), S. Ishihara (Tokyo University), K. Takahashi (Tokyo Metropolitan Cancer and Infectious Diseases Center Komagome Hospital), Y. Kanemitsu (National Cancer Center Hospital), T. Kiyomatsu (National Center for Global Health and Medicine), Y. Kinugasa, S. Yamauchi (Tokyo Medical and Dental University), K. Okabayashi (Keio University), Y. Hashiguchi (Teikyo University), T. Masaki (Kyorin University), M. Watanabe (Kitasato University), A. Shiomi (Shizuoka Cancer Center), T. Hanai (Fujita Health University), K. Komori (Aichi Cancer Center Hospital), Y. Sakai (Kyoto University), M. Ohue (Osaka International Cancer
Institute), S. Noura (Osaka Rosai Hospital), N. Tomita (Hyogo College of Medicine) and Y. Akagi (Kurume University).

\section{CONFLICTS OF INTEREST}

Authors have no conflicts of interest to declare.

\section{FUNDING}

None.

\section{REFERENCES}

1. Brierley JD, Gospodarowicz MK, Wittekind C. International Union Against Cancer (UICC). TNM Classification of Malignant Tumours, 8th ed. Oxford: John Wiley \& Sons Ltd; 2017.

2. Fortea-Sanchis C, Martínez-Ramos D, Escrig-Sos J. The lymph node status as a prognostic factor in colon cancer: comparative population study of classifications using the logarithm of the ratio between metastatic and nonmetastatic nodes (LODDS) versus the pN-TNM classification and ganglion ratio systems. BMC Cancer. 2018; 18:1208. https://doi.org/10.1186/s12885-018-5048-4. [PubMed]

3. Veen T, Nedrebø BS, Stormark K, Søreide JA, Kørner H, Søreide K. Qualitative and quantitative issues of lymph nodes as prognostic factor in colon cancer. Dig Surg. 2013; 30:1-11. https://doi.org/10.1159/000349923. [PubMed]

4. Chen SL, Bilchik AJ. More extensive nodal dissection improves survival for stages I to III of colon cancer: a population-based study. Ann Surg. 2006; 244:602-610. https:// doi.org/10.1097/01.sla.0000237655.11717.50. [PubMed]

5. Johnson PM, Porter GA, Ricciardi R, Baxter NN. Increasing negative lymph node count is independently associated with improved long-term survival in stage IIIB and IIIC colon cancer. J Clin Oncol. 2006; 24:3570-3575. https://doi. org/10.1200/JCO.2006.06.8866. [PubMed]

6. Chang GJ, Rodriguez-Bigas MA, Skibber JM, Moyer VA. Lymph node evaluation and survival after curative resection of colon cancer: systematic review. J Natl Cancer Inst. 2007; 99:433-441. https://doi.org/10.1093/jnci/djk092. [PubMed]

7. Gönen M, Schrag D, Weiser MR. Nodal staging score: a tool to assess adequate staging of node-negative colon cancer. J Clin Oncol. 2009; 27:6166-6171. https://doi. org/10.1200/JCO.2009.23.7958. [PubMed]

8. Namm J, Ng M, Roy-Chowdhury S, Morgan JW, Lum SS, Wong JH. Quantitating the impact of stage migration on staging accuracy in colorectal cancer. J Am Coll Surg. 2008; 207:882-887. https://doi.org/10.1016/j. jamcollsurg.2008.08.019. [PubMed]

9. Berger AC, Sigurdson ER, LeVoyer T, Hanlon A, Mayer RJ, Macdonald JS, Catalano PJ, Haller DG. Colon cancer survival is associated with decreasing ratio of metastatic to 
examined lymph nodes. J Clin Oncol. 2005; 23:8706-8712. https://doi.org/10.1200/JCO.2005.02.8852. [PubMed]

10. Ceelen W, Van Nieuwenhove Y, Pattyn P. Prognostic value of the lymph node ratio in stage III colorectal cancer: a systematic review. Ann Surg Oncol. 2010; 17:2847-2855. https://doi.org/10.1245/s10434-010-1158-1. [PubMed]

11. Wen J, Ye F, He X, Li S, Huang X, Xiao X, Xie X. Development and validation of a prognostic nomogram based on the log odds of positive lymph nodes (LODDS) for breast cancer. Oncotarget. 2016; 7:21046-21053. https:// doi.org/10.18632/oncotarget.8091. [PubMed]

12. Sun Z, Xu Y. Li de M, Wang ZN, Zhu GL, Huang BJ, Li $\mathrm{K}, \mathrm{Xu} \mathrm{HM}$. Log odds of positive lymph nodes: a novel prognostic indicator superior to the number-based and the ratio-based $\mathrm{N}$ category for gastric cancer patients with R0 resection. Cancer. 2010; 116:2571-2580. https://doi. org/10.1002/cncr.24989. [PubMed]

13. Calero A, Escrig-Sos J, Mingol F, Arroyo A, MartinezRamos D, de Juan M, Salvador-Sanchis JL, Garcia-Granero E, Calpena R, Lacueva FJ. Usefulness of the log odds of positive lymph nodes to predict and discriminate prognosis in gastric carcinomas. J Gastrointest Surg. 2015; 19:813820. https://doi.org/10.1007/s11605-014-2728-5. [PubMed]

14. Wang J, Hassett JM, Dayton MT, Kulaylat MN. The prognostic superiority of log odds of positive lymph nodes in stage III colon cancer. J Gastrointest Surg. 2008; 12:1790-1796. https://doi.org/10.1007/s11605-008-0651-3. [PubMed]

15. Huang B, Chen C, Ni M, Mo S, Cai G, Cai S. Log odds of positive lymph nodes is a superior prognostic indicator in stage III rectal cancer patients: A retrospective analysis of 17,632 patients in the SEER database. Int J Surg. 2016; 32:24-30. https://doi.org/10.1016/j.ijsu.2016.06.002. [PubMed]

16. MD Anderson Head and Neck Cancer Symptom Working Group. Beyond mean pharyngeal constrictor dose for beam path toxicity in non-target swallowing muscles: Dose-volume correlates of chronic radiation-associated dysphagia (RAD) after oropharyngeal intensity modulated radiotherapy. Radiother Oncol. 2016; 118:304-314. https:// doi.org/10.1016/j.radonc.2016.01.019. [PubMed]

17. Govindarajan A, Baxter NN. Lymph node evaluation in early-stage colon cancer. Clin Colorectal Cancer. 2008; 7:240-246. $\quad$ https://doi.org/10.3816/CCC.2008.n.031. [PubMed]
18. Leibl S, Tsybrovskyy O, Denk H. How many lymph nodes are necessary to stage early and advanced adenocarcinoma of the sigmoid colon and upper rectum? Virchows Arch. 2003; 443:133-138. https://doi.org/10.1007/s00428-0030858-3. [PubMed]

19. Reiman JM, Kmieciak M, Manjili MH, Knutson KL. Tumor immunoediting and immunosculpting pathways to cancer progression. Semin Cancer Biol. 2007; 17:275-287. https:// doi.org/10.1016/i.semcancer.2007.06.009. [PubMed]

20. Dougan M, Dranoff G. The immune response to tumors. Curr Protoc Immunol. 2009; Chapter 20:Unit 20.11. https:// doi.org/10.1002/0471142735.im2011s85. [PubMed]

21. Rosenberg R, Hoos A, Mueller J, Baier P, Stricker D, Werner M, Nekarda H, Siewert JR. Prognostic significance of cytokeratin-20 reverse transcriptase polymerase chain reaction in lymph nodes of node-negative colorectal cancer patients. J Clin Oncol. 2002; 20:1049-1055. https://doi. org/10.1200/JCO.2002.20.4.1049. [PubMed]

22. Park IJ, Choi GS, Jun SH. Nodal stage of stage III colon cancer: the impact of metastatic lymph node ratio. J Surg Oncol. 2009; 100:240-243. https://doi.org/10.1002/ jso.21273. [PubMed]

23. Persiani R, Cananzi FC, Biondi A, Paliani G, Tufo A, Ferrara F, Vigorita V, D'Ugo D. Log odds of positive lymph nodes in colon cancer: a meaningful ratio-based lymph node classification system. World J Surg. 2012; 36:667-674. https://doi.org/10.1007/s00268-011-1415-x. [PubMed]

24. Arslan NC, Sokmen S, Canda AE, Terzi C, Sarioglu S. The prognostic impact of the log odds of positive lymph nodes in colon cancer. Colorectal Dis. 2014; 16:O386-O392. https://doi.org/10.1111/codi.12702. [ubMed]

25. Fang HY, Yang H, He ZS, Zhao H, Fu ZM, Zhou FX, Zhou YF. Log odds of positive lymph nodes is superior to the number- and ratio-based lymph node classification systems for colorectal cancer patients undergoing curative (R0) resection. Mol Clin Oncol. 2017; 6:782-788. https://doi. org $/ 10.3892 / \mathrm{mco} .2017 .1203$. [PubMed] 